\title{
Westward Drift of the Equatorial Component of the Earth's Magnetic Dipole
}

\author{
Tsuneji RIKITAKE \\ Earthquake Research Institute, University of Tokyo \\ (Received May 30, 1966)
}

\begin{abstract}
A theory of free magneto-hydrodynamic oscillation, which gives rise to an $S_{1}{ }^{1}$ type magnetic field, superposing on the fluid dynamo in the earth's core is advanced. It is proved that an $S_{1}{ }^{1}$ field travelling eastwards relative to the core material is possible to exist. If the general westward drift of the core relative to the mantle is taken into account, the drift speed of the $S_{1}{ }^{1}$ field relative to the mantle becomes slow though still westward. The speed thus estimated roughly agrees with the observed velocity for the drift of the equatorial component of the geomagnetic dipole.
\end{abstract}

\section{Introduction}

Nagata (1965) recently emphasized a tendency of westward rotation for the equatorial component of the geomagnetic dipole although the rotation speed seems to be much smaller than that of the westward drift of the non-dipole part of the geomagnetic field. He estimated that the angular velocity amounts to only 0.05 degree/yr for the rotation of the equatorial dipole.

According to Nagata, who classified the geomagnetic secular variation into five major categories; i.e. (a) decrease in the moment of the geomagnetic dipole with a rate $0.05 \% / \mathrm{yr}$, (b) westward rotation of the equatorial component of the earth's magnetic dipole with an angular speed of 0.05 degree/yr (c) northward shift of the geomagnetic dipole with a speed of $2 \mathrm{~km} / \mathrm{yr}$, (d) westward drift of the non-dipole field with an angular speed of 0.2 degree/yr, and (e) growth and decay of the non-dipole field having a rate of 10-100 $\mathrm{r} / \mathrm{yr}$, these features of geomagnetic secular variation seem likely to be interpreted by magneto-hydrodynamic processes taking place within the earth's core in association with a dynamo action which maintains the main portion of the geomagnetic field.

A number of theories have so far been put forward in order to account for the major features of geomagnetic secular variation as reviewed by Nagata $(1962,1965)$ and Rikitake (1966). Although some of the theories are so crude that discussion was sometimes only qualitative, it has become widely supported that some sort of magneto-hydrodynamic effect would be the main cause of the secular variation. Among the five points cited in the above, however, no adequate theory on (b) has ever been tried.

In his detailed study of the westward drift of the non-dipole field, Yukutake (1962) found that the drift velocity excluding the equatorial component of the dipole amounts to 0.2 degree/yr. He also showed that, if the equatorial component of the dipole is included in the 
estimate of the drift velocity, a very small drift velocity amounting to only 0.062 degree/yr is to be obtained. On the basis of the difference in drift velocity between the two fields, he suggested that the origin of the equatorial part of the dipole might be completely different from that of the non-dipole field.

Nagata (1962) examined the westward drift velocity from the Gauss coefficients and their time-derivatives for epoch 1958.5. In Table 1 are reproduced Nagata's result which clearly indicates that the velocity for the equatorial dipole part $(n=1, m=1)$ is smaller than those for the other non-dipole field.

Table 1. Westward drift velocities in units of degree/yr obtained from the Gauss coefficients and their time-derivatives for 1958.5. (After Nagata)

\begin{tabular}{c|c|c}
\hline$n$ & $m$ & Drift velocity \\
\hline 1 & 1 & 0.055 \\
2 & 1 & 0.26 \\
2 & 2 & 0.32 \\
3 & 1 & 0.35 \\
3 & 2 & -0.083 \\
3 & 3 & 0.14 \\
4 & 1 & 0.19 \\
4 & 2 & 0.10 \\
4 & 3 & 0.17 \\
4 & 4 & 0.14 \\
\hline
\end{tabular}

Hide (1966) also summarized the drift velocity of spherical harmonic constituents for various epochs and confirmed the smallness of that of the $n=1, m=1$ component.

In the light of the above studies, it appears to the writer that the westward drift velocity of the equatorial component of the geomagnetic dipole is definitely smaller than that of the non-dipole field by a factor 3 or so. As pointed out by Nagata (1965), however, no adequate theory which accounts for the difference in drift velocity between the equatorial dipole and the non-dipole field has been put forward yet.

Rikitake (1955a, 1955 b, 1956 a, 1956 b) tried to study magneto-hydrodynamic oscillation of the axial dipole type ( $S_{1}$ field). It transpired, however, that the effect of the Coriolis force due to the earth's rotation is so strong that free oscillation would be prohibited provided a steady or quasi-steady dynamo as suggested by Bullard and Gellman (1954) is supposed to be at work in the core. Nagata and Rikitake (1963) showed, however, the possibility of magneto-hydrodynamic oscillation of the axial quadrupole type $\left(S_{2}\right)$ superimposed on the Bullard-Gellman dynamo, such an oscillation being applied to the interpretation of the northward shift of the dipole though not quite successful.

Hide (1966) studied magneto-hydrodynamic oscillations of a rotating spherical shell of an incompressible fluid pervaded by a toroidal magnetic field by means of a method similar to that for tidal oscillations in the atmosphere. He suggested that dispersive magnetohydrodynamic waves derived from the study may account for the observed secular variation 
including the westward drift.

This paper aims at extending the theory of magneto-hydrodynamic oscillation superimposed on the dynamo to the field of the equatorial dipole type $\left(S_{1}{ }^{1}\right.$ field $)$ and also at seeing whether or not a drifting field of that type is possible. If we find a drifting field, direction and speed of the drift are of great interest.

In Section 2 will be outlined the theory of magneto-hydrodynamic oscillation which more or less follows the writer's previous studies on the $S_{1}$ and $S_{2}$ fields. The theory will reduce to an eigen-value problem solving which a drift velocity of the $S_{1}{ }^{1}$ field will actually be obtained in Section 3. Section 4 will be reserved for discussion about the evaluation of the present theory.

\section{Theory}

Let us assume a steady or nearly steady dynamo as described by Bullard and Gellman (1954) in the earth's core. When a small disturbance of magnetic field is superimposed on such a system, it is governed by equation

$$
\left\{\frac{\partial}{\partial t}-(4 \pi \sigma)^{-1} \nabla^{2}\right\} \vec{h}=\operatorname{curl}\left(\vec{V}_{0} \wedge \vec{h}\right)+\operatorname{curl}\left(\vec{v} \wedge \vec{H}_{0}\right),
$$

which is reduced from Maxwell's equations on conditions that displacement current is ignored and that the magnetic permeability is unity in the electromagnetic unit. In (1), $t$ is time and $\sigma$ electrical conductivity, while $\vec{V}_{0}$ and $\vec{H}_{0}$ are the steady velocity and magnetic field of the dynamo in the core. A velocity $\vec{v}$ is set up by the interaction between $\vec{h}$ and $\overrightarrow{H_{0}}$. $\vec{h}$ and $\vec{v}$ are assumed as the first order small quantities, so that the second order quantities like $|\vec{h}| \cdot|\vec{v}|$ are ignored.

The equation of motion, which controls $\vec{v}$, can be written as

$$
\rho \frac{\partial \vec{v}}{\partial t}+2 \rho\left(\vec{\omega}_{0} \wedge \vec{v}\right)=(4 \pi)^{-1}\left(\operatorname{curl} \vec{h} \wedge \vec{H}_{0}+\operatorname{curl} \vec{H}_{0} \wedge \vec{h}\right)-\operatorname{grad} p,
$$

provided the inertia term is disregarded. $\vec{\omega}_{0}, \rho$ and $p$ denote respectively the rotation vector about the $\theta=0$ axis, density and pressure.

Assuming incompressibility of the core material and taking divergence of both the sides of (2), we obtain

$$
\nabla^{2} p=(4 \pi)^{-1}\left(\vec{H}_{0} \cdot \nabla^{2} \vec{h}+\vec{h} \cdot \nabla^{2} \vec{H}_{0}+2 \operatorname{curl} \vec{h} \cdot \operatorname{curl} \vec{H}_{0}\right)+2 \rho \vec{\omega}_{0} \cdot \operatorname{curl} \vec{v} .
$$

We are now in a position to rewrite the righthand-side of (3) in a form suitable for the present problem. Since our concern in this paper is time-dependent behaviour of the $S_{1}{ }^{1}$ magnetic field, $\vec{h}$ is assumed to be given by

$$
\vec{h}=\vec{h}_{c}+\vec{h}_{s}
$$

where

$$
\vec{h}_{c}=\left\{\begin{array}{l}
-2 s_{c} P_{1}^{1} \cos \phi \\
-\left(r \frac{\partial s_{c}}{\partial r}+2 s_{c}\right) \frac{d P_{1}{ }^{1}}{d \theta} \cos \phi
\end{array} \quad \vec{h}_{s}=\left\{\begin{array}{l}
-2 s_{s} P_{1}{ }^{1} \sin \phi \\
-\left(r \frac{\partial s_{s}}{\partial r}+2 s_{s}\right) \frac{d P_{1}{ }^{1}}{d \theta} \sin \phi
\end{array}\right.\right.
$$




$$
\left(\left(r \frac{\partial s_{c}}{\partial r}+2 s_{c}\right) \frac{P_{1}^{1}}{\sin \theta} \sin \phi, \quad \quad\left(-\left(r \frac{\partial s_{s}}{\partial r}+2 s_{s}\right) \frac{P_{1}{ }^{1}}{\sin \theta} \cos \phi,\right.\right.
$$

in which $P_{1}{ }^{1}(\cos \theta)$ is the surface spherical harmonic of degree 1 and order $1 . s_{c}$ and $s_{s}$ are generally functions of $r$ and $t . S_{n}{ }^{m}$ and $T_{n}{ }^{m}$ are in this paper used for indicating poloidal and toroidal fields specified by a spherical function having degree $n$ and order $m$. General expressions for $S_{n}{ }^{m}$ and $T_{n}{ }^{m}$ fields can be found elsewhere (i.e. Rikitake (1966)).

As for the steady magnetic field, we may assume $S_{1}, T_{2}, T_{2}{ }^{2 c}$ and $T_{2}{ }^{2 s}$ fields which appear in the simplest dynamo studied by Bullard and Gellman (1954). It has been surmised that the intensity of the $T_{2}$ field would amount to the order of 100 Gauss, that of the $S_{1}$ field to a few Gauss, and also that those of the $T_{2}{ }^{2 c}$ and $T_{2}{ }^{2 s}$ fields are intermediate. It would therefore be justified to neglect the $S_{1}, T_{2}{ }^{2 c}$ and $T_{2}{ }^{2 s}$ fields in the following estimation of the terms appearing on the righthand-side of (3). In the case of the previous discussions about the time-dependent behaviour of the $S_{1}$ and $S_{2}$ fields (Rikitake, $1955 \mathrm{a} .1956 \mathrm{~b}$, Nagata and Rikitake, 1963), however, such a neglect was not possible because the intersction between the disturbance field and the steady $S_{1}, T_{2}{ }^{2 c}$ and $T_{2}{ }^{2 s}$ fields was more important rather than the $T_{2}$ field for giving rise to motions which in turn excite the fields of the $S_{1}$ and $S_{2}$ types.

The $T_{2}$ field being written as

$$
\overrightarrow{H_{0}}=\left\{\begin{array}{l}
0 \\
0 \\
a^{-2} T_{2} r^{2} \frac{d P_{2}}{d \theta},
\end{array}\right.
$$

where $a$ is the radius of the core, $T_{2}$ is assumed to be independent of $r$ for the sake of simplicity. From (4) and (5), it is seen that all the electromagnetic interactions occurring on the righthand-side of (3) involve only $P_{2}{ }^{1} \cos \phi$ and $P_{2}{ }^{1} \sin \phi$.

The simplest dyanmo studied by Bullard and Gellman (1954) involves two types of velocities, $T_{1}$ and $S_{2}{ }^{2 c}$. Bullard and Gellman showed, however, that the velocity of the $T_{1}$ motion should be larger than that of the $S_{2}{ }^{2 c}$ motion by a foctor 10-100 in order to maintain a steady state. In view of this, we may ignore the $S_{2}{ }^{2 c}$ motion in the present discussion. In that case the steady velocity is given by

$$
\vec{V}_{0}=\left\{\begin{array}{l}
0 \\
0 \\
a^{-1} V_{1} r \frac{d P_{1}}{d \theta},
\end{array}\right.
$$

where $V_{1}$ is assumed to be independent of $r$. The interaction between the magnetic fields and the electric currents gives rise to $S_{2}{ }^{1 c}, S_{2}{ }^{1 s}, T_{3}{ }^{1 c}$ and $T_{3}{ }^{1 s}$ motions. We select only the first two which set up magnetic fields of the $S_{1}{ }^{1 c}$ and $S_{1}{ }^{1 s}$ types through the interaction with the steady magnetic field. We therefore put

$$
\vec{v}=\vec{v}_{c}+\vec{v}_{s}
$$

where 


$$
\vec{v}_{c}=\left\{\begin{array}{l}
-6 \xi_{c} r P_{2}{ }^{1} \cos \phi \\
-\left(r \frac{\partial \xi_{c}}{\partial r}+3 \xi_{c}\right) r \frac{d P_{2}{ }^{1}}{d \theta} \cos \phi \\
\left(r \frac{\partial \xi_{c}}{\partial r}+3 \xi_{c}\right) r \frac{P_{2}{ }^{1}}{\sin \theta} \sin \phi,
\end{array} \quad \vec{v}_{s}=\left\{\begin{array}{l}
-6 \xi_{s} r P_{2}{ }^{1} \sin \phi \\
-\left(r \frac{\partial \xi_{s}}{\partial r}+3 \xi_{s}\right) r \frac{d P_{2}{ }^{1}}{d \theta} \sin \phi \\
-\left(r \frac{\partial \xi_{s}}{\partial r}+3 \xi_{s}\right) r \frac{P_{2}{ }^{1}}{\sin \theta} \cos \phi
\end{array}\right.\right.
$$

From (4), (5) and (7) together with

$$
\vec{\omega}_{0}=\left\{\begin{array}{l}
\omega \cos \theta \\
-\omega \sin \theta \\
0
\end{array},\right.
$$

it is now possible to calculate the righthand-side of (3) as

$$
\nabla^{2} p=f_{c} P_{2}{ }^{1} \cos \phi+f_{s} P_{2}{ }^{1} \sin \phi,
$$

where

$$
\left.\begin{array}{l}
f_{c}=-(4 \pi)^{-1} a^{-2} r^{2} T_{2}\left(r \frac{\partial^{3} s_{s}}{\partial r^{3}}+12 \frac{\partial^{2} s_{s}}{\partial r^{2}}+\frac{28}{r} \frac{\partial s_{s}}{\partial r}\right)-2 \rho \omega r\left(r \frac{r \partial^{2} \xi_{s}}{\partial r^{2}}+6 \frac{\partial \xi_{s}}{\partial r}\right), \\
f_{s}=(4 \pi)^{-1} a^{-2} r^{2} T_{2}\left(r \frac{\partial^{3} s_{c}}{\partial r^{3}}+12 \frac{\partial^{2} s_{c}}{\partial r^{2}}+\frac{28}{r} \frac{\partial s_{c}}{\partial r}\right)+2 \rho \omega r\left(r \frac{\partial^{2} \xi_{c}}{\partial r^{2}}+6 \frac{\partial \xi_{c}}{\partial r}\right) .
\end{array}\right\}
$$

The solution of (9) that remains finite at $r=0$ is readily obtained as

$$
p=q_{c} P_{2}{ }^{2} \cos \phi+q_{s} P_{2}{ }_{2} \sin \phi,
$$

where

$$
\left.\begin{array}{l}
q_{c}=a^{-2} r^{2} K_{c}-(4 \pi)^{-1} a^{-2} T_{2}\left(r^{3} \frac{\partial s_{s}}{\partial r}+4 r^{2} s_{s}-2 r^{-3} \int r^{4} s_{s} d r\right)-2 \rho \omega r^{2} \xi_{s}, \\
q_{s}=a^{-2} r^{2} K_{s}+(4 \pi)^{-1} a^{-2} T_{2}\left(r^{3} \frac{\partial s_{c}}{\partial r}+4 r^{2} s_{c}-2 r^{-3} \int r^{4} s_{c} d r\right)+2 \rho \omega r^{2} \xi_{c},
\end{array}\right\}
$$

in which $K_{c}$ and $K_{s}$ are constants which are to be determined from the boundary conditions.

Since $p$ is obtained, the radial parts of $\vec{v}$ can be obtained from (2) as

$$
\begin{aligned}
& 3 \rho \frac{\partial \xi_{c}}{\partial t}=a^{-2} K_{c}-\frac{3}{4 \pi} a^{-2} T_{2} r^{-5} \int r^{4} s_{s} d r+\rho \omega \xi_{s}, \\
& 3 \rho \frac{\partial \xi_{s}}{\partial t}=a^{-2} K_{s}+\frac{3}{4 \pi} a^{-2} T_{2} r^{-5} \int r^{4} s_{c} d r-\rho \omega \xi_{c} .
\end{aligned}
$$

(13) can be rewritten as

$$
\left.\begin{array}{l}
3 \rho \frac{\partial}{\partial t}\left(r \frac{\partial \xi_{c}}{\partial r}+5 \xi_{c}\right)=5 a^{-2} K_{c}-\frac{3}{4 \pi} a^{-2} T_{2} s_{s}+\rho \omega\left(r \frac{\partial \xi_{s}}{\partial r}+5 \xi_{s}\right), \\
3 \rho \frac{\partial}{\partial t}\left(r \frac{\partial \xi_{s}}{\partial r}+5 \xi_{s}\right)=5 a^{-2} K_{s}+\frac{3}{4 \pi} a^{-2} T_{2} s_{c}-\rho \omega\left(r \frac{\partial \xi_{c}}{\partial r}+5 \xi_{c}\right) .
\end{array}\right\}
$$

Going back to (1), the terms on the righthand-side can be calculated on the basis of (4), (5), (6) and (7). Although $S_{3}{ }^{1 c}, S_{3}{ }^{1 s}, T_{2}{ }^{1 c}, T_{2}{ }^{1 s}, T_{3}{ }^{1 c}$ and $T_{3}{ }^{1 s}$ magnetic fields come out from $\operatorname{curl}\left(\vec{v} \wedge \vec{H}_{0}\right)$, we ignore these fields only picking up $S_{1}{ }^{1 c}$ and $S_{1}{ }^{1 s}$ fields. As the time-constant of the core is extremely large compared to that of the present concern, we may assume 
infinite conductivity for the core, so that the second term on the lefthand-side of (1) can be dropped. Equating then the radial parts of both the sides of (1), we are led to

$$
\begin{aligned}
& \frac{\partial s_{c}}{\partial t}=a^{-1} V_{1} s_{s}+\frac{27}{5} a^{-2} r^{2} T_{2} \xi_{s}, \\
& \frac{\partial s_{s}}{\partial t}=-a^{-1} V_{1} s_{c}-\frac{27}{5} a^{-2} r^{2} T_{2} \xi_{c} .
\end{aligned}
$$

(14) and (15) provide equations that govern the time-dependent behaviour or the $S_{1}{ }^{1 c}$ and $S_{1}{ }^{1 s}$ magnetic fields and the $S_{2}{ }^{1 c}$ and $S_{2}{ }^{1 s}$ motions superimposed on the steady state of the dynamo together with the boundary conditions that the normal components of velocities vanish and that the magnetic fields are continuous at the core boundary. The boundary conditions are given as

$$
\left.\begin{array}{l}
r \frac{\partial s_{c}}{\partial r}+3 s_{c}=0, \\
r \frac{\partial s_{s}}{\partial r}+3 s_{s}=0, \\
\xi_{c}=\xi_{s}=0 .
\end{array}\right\}(\text { at } r=a)
$$

In order to study an $S_{1}{ }^{1}$ magnetic field being propagated along parallel circles, we assume

$$
\begin{aligned}
& s=\left(s_{c}+i s_{s}\right) e^{i \alpha t}, \\
& \xi=\left(\xi_{c}+i \xi_{s}\right) e^{i \alpha t},
\end{aligned}
$$

where $i=\sqrt{-1}$.

In that case (14) and (15) lead to

$$
\begin{aligned}
& \rho(3 \alpha+\omega)\left(r \frac{d \xi}{d r}+5 \xi\right)=-i 5 a^{-2} K+\frac{3}{4 \pi} a^{-2} T_{2} s, \\
& \alpha s=-a^{-1} V_{1} s-\frac{27}{5} a^{-2} r^{2} T_{2} \xi
\end{aligned}
$$

where

$$
K=K_{c}+i K_{s}
$$

Meanwhile, (16) is converted to

$$
\left.\begin{array}{l}
r \frac{d s}{d r}+3 s=0, \\
\xi=0 .
\end{array}\right\}(\text { at } r=a)
$$

It is therefore seen that (18) combined with (20) provide an eigen-equation by which time-factor $\alpha$ should be determined. At this point, we take into account the condition that the time-constant of the present concern is very much larger than the time of the earth's rotation. Such a condition make us assume that $|\omega| \gg \alpha$, so that, dropping $3 \alpha$ from the first equation of (18), we obtain

$$
r \cdot \frac{d \xi}{d r}+5 \xi=C+\frac{3}{\sqrt{4 \pi \rho}} \frac{V_{A}}{\omega} a^{-2} s
$$




$$
\left(\alpha+a^{-1} V_{1}\right) s=-\frac{27}{5} \sqrt{4 \pi \rho} V_{A} a^{-2} r^{2} \xi, \quad \quad \quad
$$

where $V_{A}$ is the Alfvén velocity defined by

$$
V_{A}=T_{2} / \sqrt{4 \pi \rho}
$$

and

$$
C=-i \frac{5 a^{-2} K}{\omega} .
$$

If $s$ is eliminated from (21), we obtain

$$
r \frac{d \xi}{d r}+\left(5+\frac{81}{5} \frac{V_{A}^{2}}{a^{4} \omega \beta} r^{2}\right) \xi=C,
$$

where

$$
\beta=\alpha+a^{-1} V_{1} .
$$

Since $\xi=0$ at $r=a$, it is then obvious that

$$
a\left(\frac{d \xi}{d r}\right)_{r=a}=C .
$$

On the other hand, the first condition of (20) leads to

$$
\left(r \frac{d \xi}{d r}+5 \xi\right)_{r=a}=0
$$

which is rewritten as

$$
C+5(\xi)_{r=a}=0 .
$$

As $\xi=0$ at $r=a$, (28) leads to $C=0$. However, such a result comes from the oversimplification during the procedure of estimating interactions between the motion and the steady magnetic field. If we retain the steady $S_{1}$ field, (15) become

$$
\left.\begin{array}{l}
\frac{\partial s_{c}}{\partial t}=a^{-1} V_{1} s_{s}+\frac{9}{5} S_{1}\left(r \frac{\partial \xi_{c}}{\partial r}+5 \xi_{c}\right)+\frac{27}{5} a^{-2} r^{2} T_{2} \xi_{s}, \\
\frac{\partial s_{s}}{\partial t}=-a^{-1} V_{1} s_{c}+\frac{9}{5} S_{1}\left(r \frac{\partial \xi_{s}}{\partial r}+5 \xi_{s}\right)-\frac{27}{5} a^{-2} r^{2} T_{2} \xi_{c},
\end{array}\right\}
$$

so that the second equation of (21) reduces to

$$
\left(\alpha+a^{-1} V_{1}\right) s=\frac{9}{5} S_{1}\left(r \frac{d \xi}{d r}+5 \xi\right)-\frac{27}{5} \sqrt{4 \pi \rho} V_{A} a^{-2} r^{2} \xi
$$

Eliminating $s$, an equation

$$
(1-A) r \frac{d \xi}{d r}+\left[5(1-A)+\frac{81}{5} \frac{V_{A}^{2}}{a^{4} \omega \beta} r^{2}\right] \xi=C
$$

is obtained in place of (24), where

$$
A=\frac{27}{5} \frac{V_{A} a^{-2}}{\sqrt{4 \pi \rho} \omega \beta} S_{1} .
$$


It is therefore seen that the boundary conditions as given by (20) result in

$$
\left.\begin{array}{l}
a\left(\frac{d \xi}{d r}\right)_{r=a}=\frac{C}{1-A}, \\
\frac{9}{5} \frac{S_{1}}{\beta}\left[\left(r^{2} \frac{d^{2} \xi}{d r^{2}}\right)_{r=a}+\frac{9 C}{1-A}\right]-\frac{27}{5} \frac{C}{1-A}=0,
\end{array}\right\}
$$

which indicates that $C$ remains finite on the condition that $\left(d^{2} \xi / d r^{2}\right)_{r=a}$ is finite.

In the following, therefore, we may assume $C \neq 0$ even if the steady $S_{1}$ field is ignored for the sake of simplicity.

The solution of (24) is known to be given by

$$
\xi=C r^{-5} \mathrm{e}^{-\frac{B}{2} r^{2}} \emptyset(r)+k r^{-5} \mathrm{e}^{-\frac{B}{2} r^{2}},
$$

in which $k$ is a free constant and $\mathscr{Q}(r)$ is defined by

$$
\Phi(r)=\int r^{4} \mathrm{e}^{\frac{B}{2} r^{2}} d r,
$$

while we write as

$$
B=\frac{81}{5} \frac{V_{A}^{2}}{a^{4} \omega \beta} .
$$

The boundary conditions that $\xi=0$ and $r \frac{d s}{d r}+3 s=0$ (i.e. $r \frac{d \xi}{d r}+5 \xi=0$ as given by (27)) at $r=a$ result in the following simultaneous equations :

$$
\left.\begin{array}{l}
C a^{-5} \mathrm{e}^{-\frac{B}{2} a^{2}} \Phi(a)+k a^{-5} \mathrm{e}^{-\frac{B}{2} a^{2}}=-\frac{C}{5}, \\
C\left[1-a^{-5} \mathrm{e}^{-\frac{B}{2} a^{2}}\left(5+B a^{2}\right) \Phi(a)\right]-k a^{-5}\left(5+B a^{2}\right) \mathrm{e}^{-\frac{B}{2} a^{2}}=C .
\end{array}\right\}
$$

Eliminating $k$ from the above two equations, we obtain

$$
5+B a^{2}=0
$$

or

$$
5+\frac{81}{5} \frac{V_{A}^{2}}{a^{2} \omega \beta}=0
$$

which reduces to

$$
\alpha=-\frac{81}{25} \frac{V_{A}^{2}}{\omega a^{2}}-a^{-1} V_{1}
$$

\section{Drift of the $S_{1}{ }^{1}$ magnetic field}

Assuming $T_{2}=100$ Gauss, $V_{1}=0.02 \mathrm{~cm} / \mathrm{sec}$ and $\rho=10 \mathrm{~g} / \mathrm{cm}^{3}$, which are generally accepted for the core, $\alpha$ is calculated from (39) as

$$
\alpha=-0.85 \times 10^{-10} \mathrm{sec}^{-1},
$$

$a=3.5 \times 10^{8} \mathrm{~cm}$ and $\omega=7.5 \times 10^{-5} \mathrm{sec}^{-1}$ being taken in the estimation.

The magnetic field that is defined by the combination of $s_{c}$ and $s_{s}$ as given by the first 
equation of (17) is written as

$$
\vec{h}_{1}{ }^{1}=\mathrm{e}^{i \alpha t}\left\{\begin{array}{l}
-2\left(s_{c} \cos \phi+i s_{s} \sin \phi\right) P_{1}{ }^{1} \\
-\left(r \frac{d}{d r}+2\right)\left(s_{c} \cos \phi+i s_{s} \sin \phi\right) \frac{d P_{1}{ }^{1}}{d \theta} \\
\left(r \frac{d}{d r}+2\right)\left(s_{c} \sin \phi-i s_{s} \cos \phi\right) \frac{P_{1}{ }^{1}}{\sin \theta}
\end{array}\right.
$$

which, assuming further

$$
s_{c}=s_{s}=s_{1}{ }^{1}
$$

becomes

$$
\vec{h}_{1}{ }^{1}=\left\{\begin{array}{l}
-2 s_{1}{ }^{1} P_{1}{ }^{1} \mathrm{e}^{i(\phi+\alpha t)} \\
-\left(r \frac{d s_{1}{ }^{1}}{d r}+2 s_{1}{ }^{1}\right) \frac{d P_{1}{ }^{1}}{d \theta} \mathrm{e}^{i(\phi+\alpha t)} \\
-\left(r \frac{d s_{1}{ }^{1}}{d r}+2 s_{1}{ }^{1}\right) \frac{\partial}{\partial \phi}\left[\frac{P_{1}{ }^{1}}{\sin \theta} \mathrm{e}^{i(\phi+\alpha t)}\right] .
\end{array}\right.
$$

It is therefore seen that the $S_{1}{ }^{1}$ field moves around the core. Since $\alpha$ is negative, the motion of the field relative to the core material is eastward. If we take the westward drift of the core as revealed by the drift of the non-dipole field into account, the speed of the motion of the $S_{1}{ }^{1}$ field as seen from a man fixed to the mantle is given by $\Omega+\alpha$, where $\Omega$ is the angular velocity of the westward drift of the non-dipole field. Adopting $\Omega=0.2^{\circ} / \mathrm{yr}$ as widely accepted, $\Omega+\alpha$ is estimated as

$$
\Omega+\alpha=0.05^{\circ} / \mathrm{yr} .
$$

It is interesting that a crude theory in the above leads to a value of the westward drift of the $S_{1}{ }^{1}$ field having a velocity which has been generally accepted. Even if we assume somewhat different values for $T_{2}$ and $V_{1}$, it is unlikely that the conclusion that the $S_{1}{ }^{1} \mathrm{drift}$ velocity is one order of magnitude smaller than that of the non-dipole field would drastically be changed.

\section{Discussion and conclusion}

It is interesting to note that the theory advanced in the foregoing sections results in a westward drift of the $S_{1}{ }^{1}$ field having a velocity which approximately agrees with the observed one. Owing to the mathematical complexity, however, the theory is developed only for $n=1$ and $m=1$. It is not known, therefore, whether or not a theory similar to the present one for different $n$ and $m$ leads to a drifting field.

We have seen in Table 1 that the $S_{3}{ }^{2}$ field is drifting eastwards relative to the mantle. According to the summary of drift velocities by Hide (1966), the $S_{3}{ }^{2}$ field seems to drift eastwards for all the epochs after the beginning of this century. If it is so, it would be extremely interesting to see whether we arrive at an eastward drift of the $S_{3}{ }^{2}$ field by extending the theory to such a case.

Nothing is practically known about the drift of the $S_{1}{ }^{1}$ field during historical and geological times. Archaeomagnetic studies by Aitken and Weaver (1965) and Kawai (1964) indicate 
a westward drift of the virtual geomagnetic pole exceeding $270^{\circ}$ in longtude since around 1300 A.D. Even an estward drift of the pole during a period from 1000 A.D. to 1300 A.D. was reported by them. However, most of such drift motion of the virtual pole seems to be explained by taking the westward drift of the non-dipole field into account as has been discussed by Yukutake (1962). According to the present theory, changes in the slip velocity of the core relative to the mantle, the intensity of the $T_{2}$ field and the velocity of the $T_{1}$ motion affect the drift velocity. If it is assumed that these elements took different magnitudes some time in the past, possibility of a drastic change in the drift speed of the $S_{1}{ }^{1}$ field cannot be ruled out.

In conclusion the writer thanks Dr. T. Yukutake for his helpful discussion.

\section{References}

Aitken, M.J. and Weaver, G.H., Recent archaeomagnetic results in England. J. Geomag. Geoelectr., 17, 391-394, 1965.

Bullard, E.C. and Gellman, H., Homogeneous dynamos and terrestrial magnetism. Phil Trans. Roy. Soc. London, Ser. A, 247, 213-278, 1954.

Hide, R., Free hydromagnetic oscillations of the earth's core and the theory of the geomagnetic secular variation. Phil. Trans. Roy. Soc. London, Ser. A, 259, 615-650, 1966.

Kawai, N., Geomagnetic secular variation revealed in the baked earths in West Japan (part 1). unpublished.

Nagata, T., The main aspects of geomagnetic secular variation-westward drift and non-drifting components. Proc. Benedum Earth Magnetism Symp., 39-55, 1962.

Nagata, T., Main characteristics of recent geomagnetic secular variation, J. Geomag. Geoelectr., 17, 263-276.

Nagata, T. and Rikitake, T., The northward shifting of the geomagnetic dipole and stability of the axial magnetic quadrupole of the earth. J. Geomag. Geoelectr; 14, 213-220, 1963.

Rikitake, T., Magneto-hydrodynamic oscillations in the earth's core. Bull. Earthquake Res. Inst., 33, 1$25,1955$.

Rikitake, T., Magneto-hydrodynamic oscillations of a conducting fluid sphere in a uniform magnetic field. Bull. Earthquake Res. Inst., 33, 175-198, 1955.

Rikitake, T., Magneto-hydrodynamic oscillations of a conducting fluid sphere under the influence of the Coriolis force. Bull.Earthquake Res. Inst., 34, 139-155, 1956.

Rikitake, T., Stability of the earth's dynamo. Bull. Earthquake. Res. Inst., 34, 283-289, 1956.

Rikitake, T., Electromagnetism and the earth's interior. Elsevier Pub. Co., 1966.

Yukutake, T., The westward drift of the magnetic field of the earth. Bull. Earthquake Res. Inst., 40,1$65,1962$. 